\title{
Ecology and mating competition influence sexual dimorphism in Tanganyikan cichlids
}

\author{
Masahito Tsuboi · Alejandro Gonzalez-Voyer • Jacob Höglund • \\ Niclas Kolm
}

\begin{abstract}
Sexual selection contributes strongly to the evolution of sexual dimorphism among animal taxa. However, recent comparative analyses have shown that evolution of sexual dimorphism can be influenced by extrinsic factors like mating system and environment, and also that different types of sexual dimorphism may present distinct evolutionary pathways. Investigating the co-variation among different types of sexual dimorphism and their association with environmental factors can therefore provide important information about the mechanisms generating variation in sexual dimorphism among contemporary species. Using phylogenetic comparative analyses comparing 49 species of Tanganyikan cichlid fishes, we first investigated the pairwise relationship between three types of sexual dimorphism [size dimorphism (SSD), colour dimorphism (COD) and shape dimorphism (SHD)] and how they were related to the strength of pre- and post-copulatory sexual selection. We then investigated the influence of ecological features on sexual dimorphism. Our results showed that although SSD was associated with the overall strength of sexual selection it was not related to other types of sexual dimorphism. Also, SSD co-varied with female size and spawning habitat, suggesting a role for female adaptations to spawn in small crevices and shells influencing SSD in this group. Further, COD and SHD were positively associated and both show positive relationships with the strength of sexual selection. Finally, the level of COD and SHD was related to habitat
\end{abstract}

M. Tsuboi · A. Gonzalez-Voyer · N. Kolm (\&)

Department of Animal Ecology/Institute of Ecology and Evolution, Evolutionary Biology Centre, Uppsala University, Norbyvägen 18 D, 75236 Uppsala, Sweden

e-mail: niclas.kolm@ebc.uu.se

M. Tsuboi · J. Höglund

Department of Population Biology and Conservation Biology/Institute of Ecology and Evolution, Evolutionary Biology Centre, Uppsala University, Norbyvägen 18 D, 75236 Uppsala, Sweden

A. Gonzalez-Voyer

Department of Integrative Ecology, Estación Biológica de Doñana (EBD-CSIC), Avenida Américo Vespucio s/n, 41092 Sevilla, Spain

M. Tsuboi

Laboratory of Insect Ecology, Faculty of Agriculture, Kyoto University, Kitashirakawaoiwakecho, Sakyouku, 6068187 Kyoto, Japan 
complexity. Our results thus highlight distinct evolutionary pathways for different types of sexual dimorphism and further that ecological factors have influenced the evolution of sexual dimorphism in Tanganyikan cichlid fishes.

Keywords Sexual dimorphism - Phylogenetic principal component analysis - Habitat · Diet · Depth · Adaptive radiation

\section{Introduction}

Sexual dimorphism, here used in its broadest sense to describe any difference in morphology between males and females of the same species, is one of the most striking consequences of sexual selection among animal taxa. As such, the study of the evolutionary mechanisms leading to sexual dimorphism has been an important field in evolutionary ecology ever since the publication of Darwin's (1871) pioneering ideas around this fascinating topic.

The most common forms of sexual dimorphism towards which analyses have been targeted are sexual differences in body size (sexual size dimorphism, SSD), sexual differences in coloration (sexual colour dimorphism, COD) and sexual differences in body shape (sexual shape dimorphism, SHD) (e.g. Andersson 1994; Møller and Birkhead 1994; Owens and Hartley 1998; Dunn et al. 2001; Stuart-Fox and Ord 2004; Fairbairn et al. 2007). Evidence from comparative analyses at the species level suggests that these three types of dimorphism can sometimes be targets of distinct selection pressures. For instance, high levels of SSD are often associated with high levels of intra-sexual competition (Fairbairn 2007 and references therein), while colour dimorphism and shape dimorphism are more often generated through mate-choice mechanisms, before or after copulation (Höglund 1989; Andersson 1994; Owens and Hartley 1998; Figuerola and Green 2000; Dunn et al. 2001; Stuart-Fox and Ord 2004; Gonzalez-Voyer et al. 2008). We note that for COD and SHD, the direction of dimorphism is most often towards more conspicuous colours and shapes in males, although exceptions certainly exist as for instance in the pipefish, Syngnathus typhle, where females are more conspicuously coloured than males (Berglund et al. 1986). The pattern is very different for SSD since females in many taxa, especially in exotherms, are larger than males, most likely due to stronger fitness benefits from increased size in females due to the positive relationship between size and fecundity (e.g. Blanckenhorn 2005 and references therein). The exception to this rule often occurs in species with high levels of male-male competition or female choice for large males where males instead tend to be larger than females (Andersson 1994; Blanckenhorn 2005; Fairbairn et al. 2007).

Another important factor that may yield distinct selection pressures on different types of sexual dimorphism is the environment. For example, the environment in which visual signals are displayed can have a strong impact on the effectiveness of a particular signal (e.g. Endler 2000). Hence, it is expected that certain environmental features, for instance light conditions and background complexity, shape the selective regime acting on those signals through their effects on signal transmission (Endler 2000). Moreover, since the habitat of a given species can show dramatic temporal variation, for instance between mating- and non-mating season and during periods of parental care, it is important to consider such differences in any analysis aimed at disentangling the effect of habitat on sexual signal evolution. The sensory drive hypothesis (Endler 1992) is an interesting expansion of how the environment can affect signal evolution. This hypothesis predicts 
that environmental conditions during signal transmission and detection impact upon the evolution of signals, receptors, and signalling behaviour. Sensory biases may vary with aspects of the environment such as food, predation and light condition, influencing the direction of evolution of sexual signals. Physical and ecological properties of the environment can thus be used to predict the direction of evolution of sexual signals. Seehausen et al. (2008) recently demonstrated associations between water depth, male coloration, and female mate preference in two parapatric cichlid fish species. This finding suggests that environmental conditions can have strong effects on the evolution of male nuptial coloration. By investigating the relationship between species' environment and sexual dimorphism, one can get insight into the reasons behind the enormous variation in sexual dimorphism among contemporary taxa.

Although the effects of sexual selection on sexual dimorphism are ubiquitous, theory predicts that sexual dimorphism can also evolve through natural selection (e.g. Shine 1989; Blanckenhorn 2000, 2005), a hypothesis sometimes known as the "niche" hypothesis (e.g. Shine 1989). For instance, in species with uni-parental care, natural selection against predation can lead to the evolution of cryptic colouration or smaller size in the caring parent and thus to sexual dimorphism in colour or size (e.g. Promislow et al. 1994; Cuervo and Møller 1999; Schütz and Taborsky 2000; Doucet and Mennill 2009). This means it is important to consider not only differences in mating-specific behaviours but also potential differences in general ecology among the sexes to fully understand the factors leading to the evolution of sexual dimorphism.

Phylogenetic comparative analysis is a powerful tool for studying coevolution (Rohlf 2001) and this approach, as mentioned above, has been used previously to investigate the correlates of different types of sexual dimorphism. However, to date, few studies have simultaneously investigated the relationships between different types of sexual dimorphism and how they correspond with different ecological factors. Tanganyikan cichlid fishes form an excellent group for studying interspecific variation in sexual dimorphism because the group shows remarkable variation in the level of different types of sexual dimorphism as well as in ecology and life-histories. In this study, we investigate how different forms of sexual dimorphism in 49 species of Tanganyikan cichlid fishes are related to the strength of sexual selection and also to ecological variables in a multiple regression framework controlling for phylogeny. First, we analyze how the different forms of sexual dimorphism (SSD, COD and SHD) are inter-related and how they co-vary with intensity of sexual selection, estimated by mating system and sperm competition, common proxies of the strength of pre- and post-copulatory sexual selection (Andersson 1994; Møller and Birkhead 1994). Second, we analyze the influence of ecological factors (habitat, diet, depth, spawning site, form of care and schooling behaviour) on the different forms of sexual dimorphism to investigate how extrinsic factors may affect the evolution of sexual dimorphism.

Materials and methods

Data

We managed to collect data on all variables of interest for 49 species (Fig. 1). We followed Gonzalez-Voyer et al. (2009) and coded habitat and diet as continuous variables reflecting habitat complexity and prey motility while form of parental care (substrate guarding or mouthbrooding) was coded as a dichotomous variable. Data on depth was collected from 


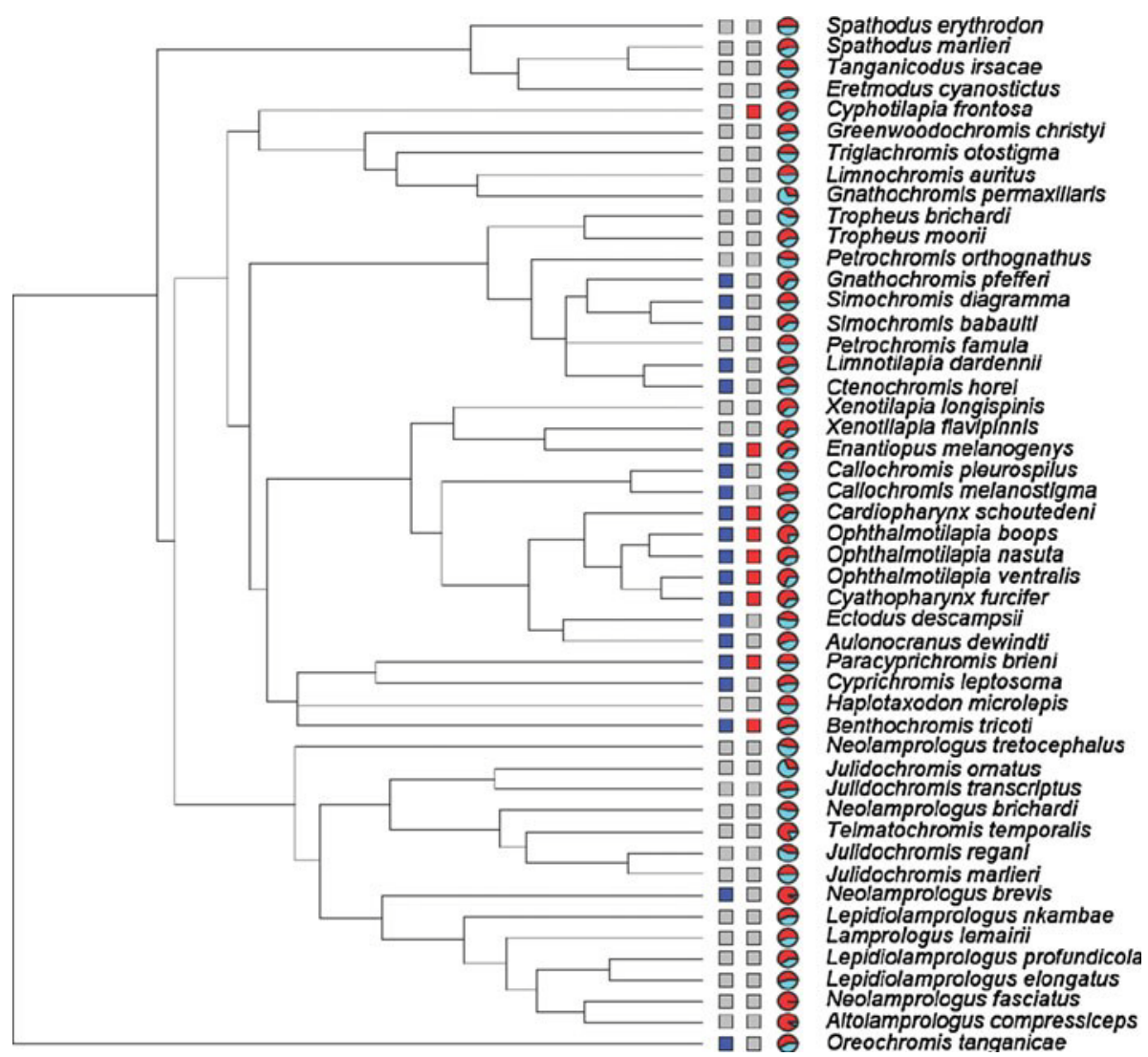

Fig. 1 Consensus phylogenetic tree of the 49 species included in the study. Shown in the figure are, from left to right: sexual dichromatism (presence dark grey squares, absence light grey squares), sexual shape dimorphism (presence dark grey squares, absence light grey squares) and finally SSD (pie charts: dark grey shows relative size of males, light grey shows relative size of female, hence monomorphic species will have a 50/50 pie-chart, male-biased species will have more dark grey, female biased species more light grey). (The phylogeny is shown as being ultrametric for illustration purposes only.)

Kohda (1991), Kuwamura (1986), Ochi (1993), Kawanabe et al. (1997), Konings (2005) and FishBase (Froese and Pauly 2010). Since information on depth was usually available as a range, we calculated the median (total range for the included 49 species: 1.5-65 meters). Schooling information was collected from Konings (2005) and coded as a dichotomous variable where species were separated into either non-schooling or schooling. Information on spawning site was collected and ranked (1-4) as: 1: water column, 2: rock side, 3: sand, 4: rock hole, 5: shell. Among the shell-breeding species, which can be facultative or obligate shell-breeders, a rank of 5 was given to both facultative and obligate shell brooders. For two species in our data-set (Altolamprologus compressiceps and Telmatochromis temporalis), there exist both rock-hole breeding and shell-breeding varieties. In both these cases our samples came from the rock-hole breeding variety, which tend to be slightly larger in overall body size, and were hence ranked as such. To ensure the ranking of these two species did not affect the results, we ran all analyses twice with these species represented as both shell-breeders and rock-hole breeders and the results remained 
qualitatively identical. Prevalence of sperm competition was ranked (1-4) following Fitzpatrick et al. (2009), based on information about mating system and fertilization location, which have previously been found to correlate strongly with different sperm characteristics (Fitzpatrick et al. 2009). Mating system was coded as in Seehausen et al. (1999) to reflect intensity of pre-copulatory sexual selection. As a measure of SSD, we adopted the size dimorphism index proposed by Lovich and Gibbons (1992): (body size of the larger sex/body size of the smaller sex) -1 . The resulting value is made negative if males are the larger sex and positive if females are the larger sex.

COD and SHD were ranked independently by four Tanganyikan cichlid experts, a similar approach to what has been used for comparative analyses of sexual dimorphism in birds (e.g. Badyaev 1997). Sexual dichromatism here represented conspicuous differences between the sexes in coloration, even if the difference was restricted to the mating period. Shape dimorphism referred to clear differences between the sexes in traits such as fins or nuchal humps, which were not only the result of differences in body size. Shape dimorphism, in this context, does not relate to less conspicuous differences between the sexes, as could be obtained using morphometric measures for example, and which could reflect ecological adaptation as well as sexual selection. For each species the judges were asked whether the sexes presented conspicuous differences in coloration or shape (independently of size dimorphism). Both variables were coded as dichotomous reflecting presence or absence of sexual differences. Disagreement between the experts was limited to sexual shape dimorphism of 4 species. In these rare occasions, we used the rank of the expert who had most experience observing the species in their natural habitat (Heinz Büscher). It is worth mentioning that cichlid fishes, including many Tanganyikan species, have a slightly different visual system than humans which allows vision also in the uv-range (Carleton 2009; O’Quin et al. 2010). Since our ranks of COD and SHD were based on human perception of these aspects of sexual dimorphism the possibility of error in the data-set cannot be ruled out. However, to limit the potential for bias, we scored COD and SHD as dichotomous variables reflecting only highly visible differences. We also note that a recent study on birds, a taxon with a similar visual system to fishes, found that human scoring of sexual dimorphism provide a valid proxy for bird perception of sexual dimorphism (Seddon et al. 2010). As such, we find it unlikely that our ranking-system of COD and SHD has affected any of our conclusions.

\section{Phylogeny}

We constructed a molecular phylogeny of the 49 Tanganyikan cichlid species under study using mitochondrial sequences downloaded from GenBank under Bayesian inference (Huelsenbeck et al. 2001) in MrBayes v3.1 (Ronquist and Huelsenbeck 2003) (See Fig. 1 for an overview of the phylogeny). We used two coding sequences, cytochrome $b$ and $\mathrm{NADH} 2$, and one non-coding gene, the control region, which were concatenated to create a matrix of 1,819 base pairs. Coding sequences were partitioned by codon and the analyses were run using a GTR ? I?c model of substitution selected using jModel test (Posada 2008). We ran 7 million iterations of the Markov chain sampling every 1,000th iteration with burnin at 1,750,000 iterations. Convergence was confirmed using AWTY (Wilgenbusch et al. 2004). Our phylogeny included species from 11 of the 16 tribes into which Tanganyikan cichlids have been classified (reviewed in Koblmüller et al. 2008), hence covering a large part of the lake's existing diversity. Branch lengths based on number of expected substitutions were included in all analyses. 
Phylogenetic generalized least-squares (PGLS) multiple regression models were used to identify the correlations between variables (Grafen 1989; Martins and Hansen 1997; Freckleton et al. 2002). PGLS-analyses were undertaken with CAIC in R (Team 2009). First, bivariate correlation models were created with variables related to sexual selection (e.g. SSD, COD, SHD, mating system, and sperm competition) and body size of both sexes independently. For these bivariate correlation-analyses we used a suite of different analyses depending on whether the traits in each correlation were discrete or continuous or both. For pairs of discrete traits we tested for an evolutionary correlation under a continuous time Markov model (Pagel 1994) in Mesquite (Maddison and Maddison 2007); for correlations between continuous and discrete traits we used a PGLS analysis with the discrete trait as independent variable, and for pairs of continuous traits we used PGLS correlation analysis. Due to the necessity of this mix of different techniques one must use caution when comparing the strength of the correlations between different pairs of traits. Secondly, we performed phylogenetically controlled multivariate models separated into two suites of analyses, one for SSD and another for COD and SHD since SSD was not related to the other two measures of sexual dimorphism (Table 1) and because both SHD and COD are most likely under inter-sexual selection based on female visual inspection. In the first of these two multivariate PGLS models, we used SSD as the dependent variable and the strength of sexual selection, all ecological and environmental variables (habitat, diet, form of care, depth, schooling and spawning habitat) and female body size as independent variables.

We used a phylogenetic principal component approach (PPCA; Revell 2009) in R (R Core Development Team 2009), with code provided by L. J. Revell, to combine our proxies of the strength of sexual selection, mating system and sperm competition, into a single principal component. Because data obtained from multiple species often violates the assumption of independence of data points, ignoring phylogenetic relationships in preliminary data transformation may result in elevated variance (Revell 2009). PPCA incorporates the expected co-variance among trait values resulting from shared ancestry into the principal component analysis (Revell 2009). From the PPCA we retained one principal component (PC) that yielded an eigenvalue over 1 (Jackson 1993) and which explained 76\% of the variation in the two variables. The loadings of this PC, i.e. the correlations with the original variables, were 0.89 for mating system and 0.85 for sperm competition. In the second model, we used visual sexual dimorphism (SHD and COD) as the dependent variable, all ecological and environmental variables (habitat, diet, form of care, depth, schooling and spawning habitat) and female body size as independent variables. Again, we used PPCA to summarize these two variables as a measure of visual sexual dimorphism in colour and shape. From this PPCA we retained one PC, that yielded an eigenvalue over 1 (Jackson 1993), which explained $71 \%$ of the variation in the two variables. The loadings of this PC, i.e. the correlations with the original variables, were -0.82 for COD and -0.82 for SHD. PGLS multiple regression models, like their non-phylogenetic counterparts, generally perform poorly when the dependent variable is dichotomous. Using a PCA-approach on a set of variables of either discrete or continuous nature is generally considered an effective way of modifying sets of variables into continuous PCA-variables when necessary for subsequent analysis (e.g. Jolliffe 2002). For each analysis, the best fit model was determined using sequential backwards removal of variables based on their P values in the full model and evaluation of each separate model by comparing their Akaike's information criterion (AIC) until the decrease of AIC between sequential models was below 2 . 
Table 1 Bivariate relationships between sexually selected traits and body size

\begin{tabular}{|c|c|c|c|c|c|c|c|c|}
\hline Traits & SSD & COD & SHD & Mating system & Sperm competition & Mean body size & Male body size & Female body size \\
\hline SSD & & $-0.09(0.18)$ & $-0.11(0.16)$ & $-0.27(0.71)$ & $-1.33(0.069)$ & $0.24(0.19)$ & $-0.08(0.63)$ & $0.52(0.0015)$ \\
\hline COD & & & ? (0.06) & $1.15(0.0013)$ & $1.15(0.0002)$ & $-0.01(0.83)$ & $0.003(0.95)$ & $-0.03(0.63)$ \\
\hline SHD & & & & $1.44(0.0013)$ & $1.31(0.0004)$ & $0.11(0.10)$ & $0.13(0.05)$ & $0.09(0.20)$ \\
\hline Mating system & & & & & $0.47(0.0002)$ & $1.32(0.17)$ & $1.43(0.14)$ & $1.05(0.23)$ \\
\hline Sperm competition & & & & & & $-0.10(0.91)$ & $0.20(0.81)$ & $-0.43(0.61)$ \\
\hline
\end{tabular}

The first number represents the non-standardised $\mathrm{b}$ for each pair of traits. Numbers within brackets represent $\mathrm{P}$ values from the PGLS or Pagel 94 correlation analysis. Statistically significant relationships at a $=0.05$ are highlighted in bold. Since the Pagel 94 analysis of correlated evolution between discrete traits does not calculate correlation coefficients, only the direction of the relationship and the $\mathrm{P}$ value is presented with ? for a positive relationship and - for a negative relationship 
Results

Bivariate correlations between variables related to sexual selection

Both COD and SHD were positively associated with both our proxies of the strength of sexual selection, mating system and the level of sperm competition (Table 1). COD was non-significantly $(\mathrm{P}=0.06)$ positively related to SHD as determined by the Pagel 94 analysis (Table 1). We note that the Pagel 94 analysis can be relatively weak in statistical power when certain states of a trait are rare (own observations), such as SHD in this dataset, and we therefore suggest that this non-significant trend really should be considered as biologically relevant. This is further supported by the strong relationship between COD and SHD in the PPCA-analysis which made combining the traits in one PC necessary to decrease the number of inferential tests and avoid problems with collinearity. Interestingly, SSD was not related to any other measure of sexual dimorphism, and only presented a nonsignificantly negative association with the level of sperm competition $(P=0.069)$. SSD was noticeably biased towards male-biased SSD (Fig. 2) and also significantly positively associated with female body size (Table 1). Closer examination of the relationship between SSD and female body size showed it was most likely driven by a low number of species with high levels of male-biased SSD and very small female size which were shell-breeders or had shell-breeding varieties (Fig. 2). We also found a strong positive association between SSD and mating system and the level of sperm competition (Table 1). Finally, we detected a non-significant positive relationship between SHD and male body size $(\mathrm{P}=0.05)$.

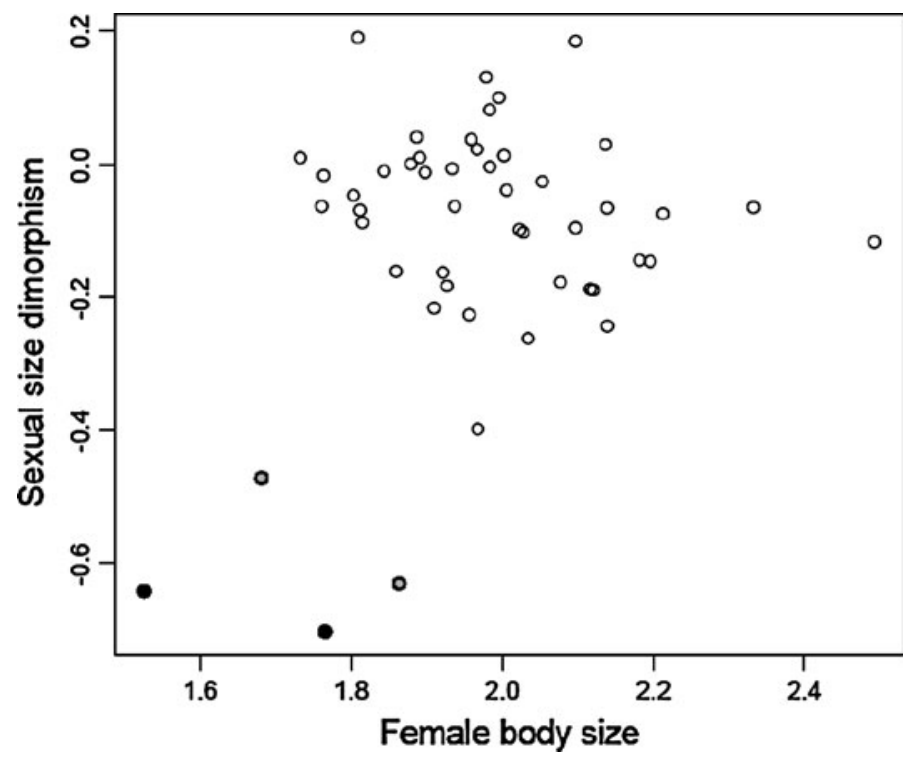

Fig. 2 Graphical plot of the relationship between female body size and sexual size dimorphism, highlighting the potential significance of the included shell-breeding species. Marked data-points represent species with shell-breeding varieties, possibly sub-species or even separate species (grey points) and obligate or facultative shell breeders (black points). Both variables are presented by log-transformed values in the figure. See text for details 
Multivariate analyses

In our multivariate analysis of how SSD was related to the strength of sexual selection and ecology, we detected associations with sexual selection and spawning site (Table 2). Based on the factor loadings of the PPCA on mating system and level of sperm competition, this indicates that species under high levels of both pre- (more promiscuous mating system) and post-copulatory sexual selection (higher possibility for sperm competition) and species breeding in more constrained spaces (e.g. crevices and shells) show higher levels of malebiased SSD. The link between spawning site and SSD further indicates that female dwarfism may have affected SSD in this group. In our multivariate analysis of how visual sexual dimorphism was related to the strength of sexual selection and ecological variables, we found associations with our proxies of the strength of sexual selection and habitat (Table 3). Based on the factor loadings of the PPCA on COD and SHD, this means that species are more sexually dimorphic, both in colour and shape, under higher levels of preand post-copulatory sexual selection. For the ecological variable habitat, the factor loadings from the PPCA translate into more sexually dimorphic species occurring in less complex habitats. None of the other variables (diet, schooling behaviour, depth, spawning habitat or form of care) were found to be statistically associated with the level of sexual dimorphism.

Table 2 Results from complete best fitted PGLS multiple regression analysis with SSD as dependent variable and strength of sexual selection and ecological factors as independent variables

\begin{tabular}{lrcc}
\hline Variable & \multicolumn{1}{l}{ b } & S.E. & P value \\
\hline Sexual selection & -0.06 & 0.02 & 0.005 \\
Depth & 0.09 & 0.08 & 0.26 \\
Diet & -0.04 & 0.02 & 0.14 \\
Spawning site & -0.06 & 0.03 & 0.047 \\
Female size & 0.21 & 0.17 & 0.21
\end{tabular}

The evolutionary parameter for the complete best fitted GLS-model, $\mathrm{k}=7 \mathbf{9} 10^{-5}$ and the complete best fitted model multiple $\mathrm{R}^{2}=0.30$. We present the partial regression coefficients (b) and their standard errors (S.E.) as well as the separate P values for each independent variable. Significant relationships at a $=0.05$ are highlighted in bold. See text for details on variables

Table 3 Results from complete best fitted PGLS multiple regression analysis with sexual dimorphism as dependent variable and strength of sexual selection and ecological factors as independent variables

\begin{tabular}{lccc}
\hline Variable & b & S.E. & P value \\
\hline Sexual selection & -0.44 & 0.07 & $<0.0001$ \\
Habitat & 0.18 & 0.04 & 0.019 \\
Spawning site & 0.14 & 0.12 & 0.23 \\
\hline
\end{tabular}

The evolutionary parameter for the complete best fitted GLS-model, $\mathrm{k}=0.49$ and the complete best fitted model multiple $\mathrm{R}^{2}=0.52$. We present the partial regression coefficients (b) and their standard errors (S.E.) as well as the separate $\mathrm{P}$ values for each independent variable. Significant relationships at $\mathrm{a}=0.05$ are highlighted in bold. See text for details on variables 
Discussion

We found positive associations between our different measures of sexual dimorphism except for SSD, which instead was related to female body size, and thus support a possibility for distinct evolution of different forms of sexual dimorphism. Our bivariate and multivariate analyses of the link between SSD and ecology and sexual selection detected no effect of any of the ecological variables but did indicate that species tended to be more sexually dimorphic (i.e. males larger than females) under stronger pre- and post-copulatory levels of sexual selection. For COD and SHD, we found higher levels of sexual dimorphism under stronger sexual selection but also an association with habitat complexity which suggest a link between the ecological setting and the potential for evolution of sexual dimorphism in this group.

That SSD stands out from our other measures of sexual dimorphism suggests that size differences among the sexes really do evolve under different selection pressures than COD and SHD. In our multivariate analysis, SSD was positively associated with both mating system and level of sperm competition but significantly associated only with spawning habitat out of our variables describing variation in ecology and parental care. Our results thus suggest a key-role for sexual selection driving SSD in this group. Although we were not able to analyse how aggression levels vary among the included cichlid species due to lack of such data, we believe that the lack of any relationship between SSD and the other types of sexual dimorphism is caused by SSD having evolved via male-male competition while the other measures of dimorphism are more likely to have evolved under female mate-choice. We base this hypothesis on several arguments. Tanganyikan cichlids are often highly territorial (e.g. Kohda 1995, 1997; Barlow 2000) and males generally defend several types of territories, for instance nesting and mating territories, in comparison to females who tend to defend only feeding territories when they do not care for offspring (Kohda 1995). Pure male-male aggressiveness is also very common (e.g. Kohda 1995, 1998; Barlow 2000) and aggression-levels have been found to be a determinant of reproductive success in this group (e.g. Rossiter and Yamagishi 1997) as well as in many other vertebrates (Andersson 1994). Since body size is strongly linked to dominance status in most vertebrates (e.g. Shine 1989; Fairbairn et al. 2007 and references therein) and also in cichlid fishes (e.g. Nelissen 1992; Kohda 1998; Barlow 2000), we thus find it very likely that male-male competition has driven SSD also in our sample of Tanganyikan cichlid species.

Many comparative analyses, over a range of taxa, have detected a positive association between the level of male biased SSD and overall species body size (e.g. Rensch 1950; Abouheif and Fairbairn 1997; Blanckenhorn 2005; Fairbairn et al. 2007). However, we did not detect such a pattern for our sample of Tanganyikan cichlids. Instead, we found that male biased SSD increased with decreasing female size (Table 1; Fig. 2). And in our multivariate analysis, we detected an association between SSD and spawning site. We suggest that these patterns at least partly have been driven by female dwarfism among species where brooding takes place in smaller crevices or shells. For instance, the shellbreeding cichlids often have extreme SSD with remarkably small females in relation to males. In fact, the highest reported male-biased SSD in any animal is found in a Tanganyikan cichlid, Lamprologus callipterus, where larger males with higher reproductive success are able to carry shells to their territories in which females brood the eggs resulting in males being [12 times larger than females (Sato and Gashagaza 1997; Schütz and Taborsky 2000; Schütz et al. 2006). As such, we propose that a combination of sexual selection and natural selection have caused the sometimes extreme levels of SSD, at least 
in these species (see also Schütz et al. 2006 for a more exhaustive discussion on this topic) and this highlights the need to consider many selective pressures to fully explain the causes of SSD (Blanckenhorn 2005). We must note that some species with ample SSD in this group (including L. callipterus) were not included in the present analyses due to lack of data for other traits of interest in the present study. However, we find it interesting that even without these species in our data-set, we still detected the above mentioned associations. Alternative, yet not mutually exclusive explanations to why male-biased SSD is higher in species breeding in more closed spawning habitats are i) that such habitats may be rarer and therefore generate strong selection on male body size in order to defend such rare resources and ii) that more closed spawning sites indeed are more easily defendable resources unlike the open water column or open rock-sides which creates the opportunity for stronger male-male competition and selection on male size. As both these alternative explanations are based on male-male competition, they extend our initial argument of sexual selection as the main mechanism driving SSD in the Tanganyikan cichlids.

The strong relationship between our proxies of sexual selection and COD and SHD supports that also these types of sexual dimorphism are mainly driven by sexual selection. As mentioned earlier, we support the view that these types of dimorphism are under selection from mate choice rather than direct male-male competition. This is likely because in most of the Tanganyikan species with elaborate male ornamentation in colour or shape, these ornaments are displayed towards females during courtship (e.g. Konings 1988). And colour dimorphism is often most extreme at the time of spawning, as for instance in Enantiopus melanogenys, where the males develop strongly-coloured orange cheeks only during courtship and spawning (Konings 1988). For SHD, the males in the dimorphic species mostly have elongated fins but sometimes also a hump-shaped head formation like in Cyphotilapia frontosa, where the dorsal muscle extends forward creating a nuchal hump that is much larger in males than in females (e.g. Konings 1988). In this species, males are reported to be non-territorial suggesting male-male competition plays little role behind the evolution of this interesting shape dimorphism. In species where males have more elongated fins, these fins often play an important role during courtship and spawning (Konings 1988). For instance, in many species with female mouthbrooding, the pelvic and anal fins are often ornamented with colourful spots that mimic eggs in shape and colour and are believed to act as egg-dummies. These egg dummies are used both to attract females and to aid in egg fertilization (Wickler 1962; Mrowka 1987; Hert 1989). Studies on other taxa, mainly birds (e.g. Møller and Birkhead 1994; Owens and Hartley 1998; Dunn et al. 2001), have previously found links between the level of plumage dimorphism and both mating system and level of sperm competition even though the link between mating system and dimorphism is far from clear (Badyaev \& Hill 2003). Our results thus strengthen the case that the intensity of sexual selection, through mating system and potentially also postcopulatory mate choice, is an important factor behind broad-scale patterns of sexual dimorphism across taxa. More analyses focusing on the detailed mechanistics behind this relationship would be an interesting avenue for future research.

The only factor apart from sexual selection that was associated with COD and SHD in our analyses was habitat complexity. We find it interesting that this relationship was negative, i.e. species were more sexually dimorphic in less complex habitats. Similar analyses for instance on birds and lizards have detected the opposite pattern and have suggested that more complex and/or more closed habitats select for more pronounced sexual dimorphism enabling male signals to better stand out from the surroundings (Endler 1993; Endler and Théry 1996; McNaught and Owens 2002; Stuart-Fox and Ord 2004). So why are Tanganyikan cichlids more dimorphic in less complex habitats? One possible 
explanation lies in the visual system of these fishes. A recent study comparing visual acuity among three species of Tanganyikan cichlids, from differently complex habitats at similar depths, found that visual acuity was greater among the species from the more complex habitats (Dobberfuhl et al. 2005). The authors hypothesised that this was caused by the more complex, rocky habitat, placing higher demands on spatial resolution and navigation, while the simpler sandy habitat instead places higher demands on detection of movement to avoid predators (Dobberfuhl et al. 2005). We speculate that the higher levels of sexual dimorphism in simpler habitats in our sample can be derived through two different, not necessarily mutually exclusive, mechanisms. Firstly, if lower visual acuity really is a general pattern among species in simpler habitats it could mean that stronger ornamental signals, leading to higher levels of dimorphism, are required to elicit a response in the opposite sex. Interestingly, this hypothesis is partly supported by a recent comparative analysis investigating the link between brain structure volume and ecology in Tanganyikan cichlids. This study found that the telencephalon, the brain structure processing much of the information from the surrounding environment, for instance regarding spatial cognition and learning, is in fact larger in species in more complex habitats, while no association was detected between the optic tectum, the brain structure that receives visual information, and habitat (Gonzalez-Voyer and Kolm 2010). More information is required on the link between brain morphology, visual acuity and habitat complexity before this can be fully understood. Secondly, many of the species in the simpler habitats have highly advanced courtship displays, often based on courtship on or near visual structures, e.g. crater-nests (Konings 1988; Yanagisawa et al. 1997; Rossiter and Yamagishi 1997). The strong level of sexual signalling in these species may therefore translate into high levels of habitat-specific dimorphism. None of the other variables related to ecology were found to be associated to the level of COD and SHD. We find this surprising, especially regarding depth which has been found to play an important role in the evolution of colouration in other cichlids (e.g. Seehausen et al. 2008). We note that our study focused on sexual dimorphism only and that analysis of overall colouration in the separate sexes, which could well be associated to the different lighting conditions at different depths, is beyond the scope of the present study.

To conclude, we found support for distinct evolution of size dimorphism in relation to dimorphism in colour and shape and further that variation in sexual dimorphism was associated with the strength of sexual selection and at least one ecological variable: habitat complexity. Future studies that examine these patterns in even greater detail, for instance with data on behavioural variation and the neural system, will be important to fully understand the evolution of sexual dimorphism under influence of the social and physical environment.

Acknowledgments This study was funded by grants from the Swedish Research Council (VR) and the Wenner-Grens Foundations to Niclas Kolm. A. Gonzalez-Voyer was funded by a Wenner-Grens Foundations post-doctoral stipend and a Juan de la Cierva post-doctoral contract from the Spanish Ministry of Science and Innovation. We are especially grateful to Heinz Büscher, Ola Svensson, Carl Westholm and John Fitzpatrick for their help with scoring species for sexual dimorphism in colour and shape.

\section{References}

Abouheif E, Fairbairn DJ (1997) A comparative analysis of allometry for sexual size dimorphism: assessing Rensch's rule. Am Nat 149:540-562

Andersson M (1994) Sexual selection. Princeton University Press, Princeton, NJ 
Badyaev AV (1997) Altitudinal variation in sexual dimorphism: a new pattern and alternative hypotheses. Behav Ecol 8:675-690

Badyaev AV, Hill GE (2003) Avian sexual dichromatism in relation to phylogeny and ecology. Annu Rev Ecol Evol Syst 34:27-49

Barlow GW (2000) Cichlid fishes. Nature’s grand experiment in evolution. Perseus Books, Cambridge

Berglund A, Rosenquist G, Svensson I (1986) Mate choice, fecundity and sexual dimorphism in two pipefish species (Syngnathidae). Behav Ecol Sociobiol 19:301-307

Blanckenhorn WU (2000) The evolution of body size: what keeps organisms small? Q Rev Biol 75:385-407

Blanckenhorn WU (2005) Behavioral causes and consequences of sexual size dimorphism. Ethology 111:977-1016

Carleton K (2009) Cichlid fish visual systems: mechanisms of spectral tuning. Int Zool 4:75-86

Cuervo JJ, Møller AP (1999) Ecology and evolution of extravagant feather ornaments. J Evol Biol 12:986-998

Darwin C (1871) The descent of man and selection in relation to sex. John Murray, London

Dobberfuhl AP, Ullmann JFP, Shumway CA (2005) Visual acuity, environmental complexity, and social organization in African cichlid fishes. Behav Neurosci 119:1648-1655

Doucet SM, Mennill DJ (2009) Dynamic sexual dichromatism in an explosively breeding Neotropical toad. Biol Lett 6:63-66

Dunn PO, Whittingham LA, Pitcher TE (2001) Mating system, sperm competition, and the evolution of sexual dimorphism in birds. Evolution 55:161-175

Endler JA (1992) Signals, signal conditions, and the direction of evolution. Am Nat 139:S125-S153

Endler JA (1993) Some general comments on the evolution and design of animal communication systems. Phil Trans R Soc Lond B 340:215-225

Endler JA (2000) Evolutionary implications of the interaction between animal signals and the environment. In: Espmark Y, Amundsen T, Rosenqvist G (eds) Animal signals. Tapir Academic Press, Trondheim, pp 11-46

Endler JA, Théry M (1996) Interacting effects of lek placement, display behavior, ambient light, and colour patterns in three Neotropical forest-dwelling birds. Am Nat 148:421-452

Fairbairn DJ (2007) The enigma of sexual size dimorphism. In: Blackenhorn W, Fairbairn D, Szekely T (eds) Sex, size and gender roles. Oxford University Press, Oxford, pp 27-37

Fairbairn DJ, Blanckenhorn WU, Székely T (eds) (2007) Sex, size and gender roles: evolutionary studies of sexual size dimorphism. Oxford University Press, New York

Figuerola J, Green AJ (2000) The evolution of sexual dimorphism in relation to mating patterns, cavity nesting, insularity and sympatry in the Anseriformes. Funct Ecol 14:701-710

Fitzpatrick JL, Montgomerie RM, Desjardins JK, Stiver KA, Kolm N, Balshine S (2009) Female promiscuity promotes the evolution of faster sperm in cichlid fishes. Proc Natl Acad Sci USA 106:1128-1132

Freckleton RP, Harvey PH, Pagel M (2002) Phylogenetic analysis and comparative data: a test and review of evidence. Am Nat 160:712-726

Froese R, Pauly D (2010) FishBase. World Wide Web electronic publication. www.fishbase.org, version $(11 / 2010)$

Gonzalez-Voyer A, Kolm N (2010) Sex, ecology and the brain: evolutionary correlates of brain structure volumes in Tanganyikan cichlids. PLoS ONE 5(12):e14355

Gonzalez-Voyer A, Fitzpatrick JL, Kolm N (2008) Sexual selection determines parental care patterns in cichlid fishes. Evolution 62:2015-2026

Gonzalez-Voyer A, Winberg S, Kolm N (2009) Social fishes and single mothers: brain evolution in African cichlids. Proc R Soc Lond B 276:161-167

Grafen A (1989) The phylogenetic regression. Phil Trans R Soc Lond B 326:119-157

Hert E (1989) The function of egg-spots in an African mouth-brooding cichlid fish. Anim Behav 37:726-732

Höglund J (1989) Size and plumage dimorphism in lek-breeding birds: a comparative analysis. Am Nat 134:72-87

Huelsenbeck JP, Ronquist F, Nielsen R, Bollback JP (2001) Bayesian inference of phylogeny and its impact on evolutionary biology. Science 294:2310-2314

Jackson D (1993) Stopping rules in principal components analysis: a comparison of heuristical and statistical approaches. Ecology 74:2204-2214

Jolliffe IT (2002) Principal component analysis, 2nd edn. Springer, New-York

Kawanabe H, Hori M, Nagoshi M (1997) Fish communities in Lake Tanganyika. Kyoto University Press, Kyoto

Koblmüller S, Sefc KM, Sturmbauer C (2008) The lake Tanganyika cichlid species assemblage: recent advances in molecular phylogenetics. Fourth symposium on speciation in Ancient Lakes, Berlin, Germany, Hydrobiologia 
Kohda M (1991) Intra- and interspecific social organization among three herbivorous cichlid fishes in Lake Tanganyika. Japan J Ichtyol 38:147-163

Kohda M (1995) Territoriality of male cichlid fishes in Lake Tanganyika. Ecol Freshw Fish 4:180-184

Kohda M (1997) Interspecific society among herbivorous cichlid fishes. In: Kawanabe H, Hori M, Nagoshi M (eds) Fish communities in Lake Tanganyika. Kyoto University Press, Kyoto, pp 105-117

Kohda M (1998) Coexistence of permanently territorial cichlids of the genus Petrochromis through malemating attack. Env Biol Fish 52:231-242

Konings A (1988) Tanganyika cichlids. Verdujin Cichlids, Zevenhuizen

Konings A (2005) Back to nature guide to Tanganyika cichlids, 2nd edn. Cichlid Press, St. Leon-Rot

Kuwamura T (1986) Parental care and mating systems of cichlid fishes in Lake Tanganyika: a preliminary field survey. J Ethol 4:129-146

Lovich JE, Gibbons JW (1992) A review of techniques for quantifying sexual size dimorphism. Growth Dev Aging 56:269-281

Maddison WP, Maddison DR (2007) Mesquite: a modular system for evolutionary analysis. Version 2.7. http://mesquiteproject.org

Martins EP, Hansen TF (1997) Phylogenies and the comparative method: a general approach to incorporating phylogenetic information into the analysis of interspecific data. Am Nat 149:646-667

McNaught MK, Owens IPF (2002) Interspecific variation in plumage colour among birds: species recognition or light environment? J Evol Biol 15:505-514

Møller AP, Birkhead TR (1994) The evolution of plumage brightness in birds is related to extrapair paternity. Evolution 48:1089-1100

Mrowka W (1987) Oral fertilization in a mouth-brooding cichlid fish. Ethology 74:293-296

Nelissen MHJ (1992) Does body size affect the rank of a cichlid fish in a dominance hierarchy? J Ethol 10:153-156

$\mathrm{O}^{\circ}$ Quin KE, Hofmann CM, Hofmann HA, Carleton KL (2010) Parallel evolution of opsin gene expression in African Cichlid fishes. Mol Biol Evol 27:2839-2854

Ochi H (1993) Mate monopolization by a dominant male in a multi-male social group of a mouthbrooding cichlid, Ctenochromis horei. Japan J Ichtyol 40:209-218

Owens IPF, Hartley IR (1998) Sexual dimorphism in birds: why are there so many different forms of dimorphism? Proc R Soc Lond B 265:397-407

Pagel MD (1994) Detecting correlated evolution on phylogenies: a general method for the comparative analysis of discrete characters. Proc R Soc Lond B 255:37-45

Posada D (2008) jModelTest: phylogenetic model averaging. Mol Biol Evol 25:1253-1256

Promislow D, Montgomerie R, Martin TE (1994) Sexual selection and survival in North-American waterfowl. Evolution 48:2045-2050

R Core Development Team (2009) R: a language and environment for statistical computing. R Foundation for Statistical Computing, Vienna, Austria

Rensch B (1950) Die abhangigkeit der relativen sexualdifferenz von der Körpergroße. Bonner Zool Beiträge 1:58-69

Revell LJ (2009) Size-correction and principal components for interspecific comparative studies. Evolution 63:3258-3268

Rohlf FJ (2001) Comparative methods for the analysis of continuous variables: geometric interpretations. Evolution 55:2143-2160

Ronquist F, Huelsenbeck JP (2003) MrBayes 3: Bayesian phylognetic inference under mixed models. Bioinformatics 19:1572-1574

Rossiter A, Yamagishi S (1997) Intraspecific plasticity in the social system and mating behaviour of a lekbreeding cichlid fish. In: Kawanabe H, Hori M, Nagoshi M (eds) Fish communities in Lake Tanganyika. Kyoto University Press, Kyoto, pp 193-217

Sato T, Gashagaza MM (1997) Shell-brooding cichlid fishes of Lake Tanganyika: their habitats and mating systems. Kyoto University Press, Kyoto

Schütz D, Taborsky M (2000) Giant males or dwarf females: what determines the extreme sexual size dimorphism in Lamprologus callipterus? J Fish Biol 57:1254-1265

Schütz D, Parker GA, Taborsky M, Sato T (2006) An optimality approach to male and female body sizes in an extremely size-dimorphic cichlid fish. Evol Ecol Res 8:1-16

Seddon N, Tobias JA, Eaton M, Ödeen A, Byers BE (2010) Human vision can provide a valid proxy for avian perception of sexual dichromatism. Auk 127:283-292

Seehausen O, Mayhew PJ, Van Alphen JJM (1999) Evolution of colour patterns in East African cichlid fish. J Evol Biol 12:514-534 
Seehausen O, Terai Y, Magalhaes IS, Carleton KL, Mrosso HDJ, Miyagi R, van der Sluijs I, Schneider MV, Maan ME, Tachida H, Imai H, Okada N (2008) Speciation through sensory drive in cichlid fish. Nature 455:620-626

Shine R (1989) Ecological causes for the evolution of sexual size dimorphism: a review of the evidence. Q Rev Biol 64:419-461

Stuart-Fox DM, Ord TJ (2004) Sexual selection, natural selection and the evolution of dimorphic coloration and ornamentation in agamid lizards. Proc R Soc Lond B 271:2249-2255

Wickler W (1962) 'Egg-dummies' as natural releasers in mouth-breeding cichlids. Nature 194:1092-1094

Wilgenbusch JC, Warren DL, Swofford DL (2004) AWTY: a system for graphical exploration of MCMC convergence in Bayesian phylogenetic inference http://ceb.csit.fsu.edu/awty

Yanagisawa Y, Ochi H, Gashagaza MM (1997) Habitat use in cichlid fishes for breeding. In: Kawanabe H, Hori M, Nagoshi M (eds) Fish communities in Lake Tanganyika. Kyoto University Press, pp 151-173 